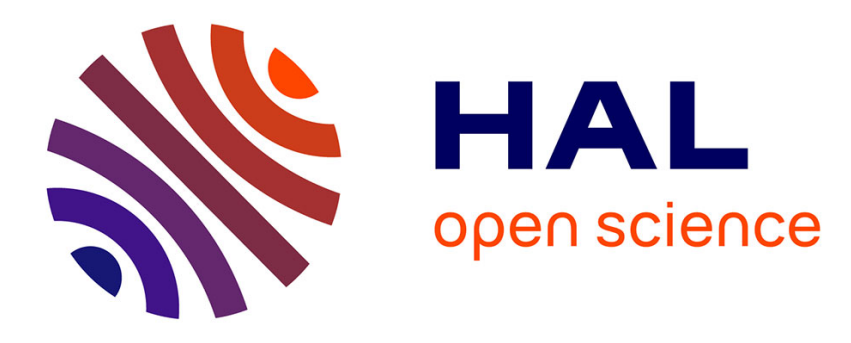

\title{
Photoélectrochimie sur électrode de phosphure de gallium de type $p$
}

\author{
A. Bourrasse, G. Horowitz
}

\section{To cite this version:}

A. Bourrasse, G. Horowitz. Photoélectrochimie sur électrode de phosphure de gallium de type p. Journal de Physique Lettres, 1977, 38 (14), pp.291-293. 10.1051/jphyslet:019770038014029100 jpa00231379

\section{HAL Id: jpa-00231379 https://hal.science/jpa-00231379}

Submitted on 1 Jan 1977

HAL is a multi-disciplinary open access archive for the deposit and dissemination of scientific research documents, whether they are published or not. The documents may come from teaching and research institutions in France or abroad, or from public or private research centers.
L'archive ouverte pluridisciplinaire HAL, est destinée au dépôt et à la diffusion de documents scientifiques de niveau recherche, publiés ou non, émanant des établissements d'enseignement et de recherche français ou étrangers, des laboratoires publics ou privés. 
Classification

Physics Abstracts

$82.45-82.50$

\title{
PHOTOÉLECTROCHIMIE SUR ÉLECTRODE DE PHOSPHURE DE GALLIUM DE TYPE $p$
}

\author{
A. BOURRASSE et G. HOROWITZ \\ Centre d'Etudes Nucléaires de Saclay, Service d'Etudes Energétiques \\ B.P. 2, 91190 Gif sur Yvette, France
}

(Reçu le 4 mars 1977, accepté le 21 juin 1977)

\begin{abstract}
Résumé. - On a étudié la possibilité d'utiliser une électrode en phosphure de gallium de type $\mathrm{p}$ comme cathode d'une cellule de photoélectrochimie. Son rendement maximum avoisine $60 \%$ et le maximum de la photoréponse est situé à $4100 \AA$. La corrosion ne devient visible que sous de très forts éclairements après plusieurs heures de fonctionnement. Cependant, cette électrode ne convient pas pour une conversion optimale de l'énergie solaire, car le maximum de sa photoréponse est localisé à la limite du bleu du spectre solaire.
\end{abstract}

\begin{abstract}
A p type gallium phosphide electrode has been studied as the cathode of a photoelectrochemical cell. Its maximum efficiency has been found to be near 0.6 and the maximum photoresponse occurs at $4100 \AA$. Corrosion appears only under very high illuminations after several hours, of operation. However this electrode is not suitable for optimal solar energy conversion because its maximum photoresponse is located at the blue edge of the solar spectrum.
\end{abstract}

1. Introduction. - Le phosphure de gallium GaP de type $\mathrm{p}$ dopé au zinc a une largeur de bande interdite de $2,25 \mathrm{eV}$ et à ce titre peut être excité par de la lumière visible lors d'expériences de photoélectrochimie.

D'après Beckmann et Memming [1], si le GaP n polarisé anodiquement ne produit aucun courant, le GaP p dans les mêmes conditions échange des charges avec l'électrolyte (système redox) par sa bande de valence et se dissout. En polarisation cathodique, le GaP n produit toujours du courant alors que le GaP p n'en produit que s'il est éclairé. L'échange des charges se fait alors par la bande de conduction et l'effet de l'éclairage est plus intense en milieu acide qu'en milieu basique. Yoneyama et al. [2] se fondant sur les travaux de Gerischer [3] a réalisé une cellule photoélectrochimique comportant une anode de $\mathrm{TiO}_{2} \mathrm{n}$ et une cathode de $\mathrm{GaP} \mathrm{p}$ plongées dans un électrolyte qui était soit du $\mathrm{SO}_{4} \mathrm{H}_{2} 1 \mathrm{~N}$, soit $\mathrm{NaOH} 1 \mathrm{~N}$. Il a ainsi obtenu la décomposition de l'eau, mais signale des détériorations des performances de la cellule au cours du temps.

L'étude que nous présentons montre le comportement d'une électrode de GaP p, sa stabilité électrique, son rendement de conversion d'énergie lumineuse en énergie électrique et sa tenue à la corrosion vis-à-vis de divers électrolytes.

2. Techniques expérimentales. - L'électrode est taillée dans un monocristal de GaP p dopé au zinc commercialisé par MCP ELECTRONICS Ltd (con- centration d'impuretés dopantes $10^{18} \mathrm{~cm}^{-3}$ ). L'orientation de la surface active est $\langle 111\rangle$ (structure cristalline zinc-blende). Le contact électrique sur la face arrière est réalisé par une évaporation d'alliage zinc-indium. Tout l'échantillon est enrobé dans une matière plastique isolante à l'exception de la surface active polie.

Le montage de l'électrode dans le dispositif d'électrolyse est un montage potentiostatique classique (Fig. 1), les tensions sont données par rapport à une électrode de référence au calomel (S.C.E.), l'éclairage est fourni par des lampes au Xe ou au $\mathrm{Hg}$ haute pression de $500 \mathrm{~W}$. La puissance lumineuse reçue par le monocristal est mesurée à l'aide d'une cellule UDT PIN $10 \mathrm{UV}$.

3. Résultats et discussions. - Caractéristiques intensité-potentiel. La figure 2 montre les caractéristiques obtenues dans des solutions de $\mathrm{SO}_{4} \mathrm{H}_{2} 1 \mathrm{~N}$ ou $\mathrm{HCl} 1 \mathrm{~N}$, l'une dans le noir, l'autre sous un éclairement monochromatique $(3800 \AA)$ de $13 \mathrm{~mW} / \mathrm{cm}^{2}$. A ce niveau d'éclairement, la reproductibilité des courbes est médiocre et dépend assez fortement des traitements de surface. Par contre, la stabilité électrique en milieu sulfurique et en milieu chlorhydrique est meilleure que celle observée par Yoneyama. Après 30 minutes de polarisation à $-1,5 V_{\text {sce }}$, l'intensité du courant n'a pratiquement pas varié. Lorsque le cristal est éclairé directement par la lumière émise par la lampe à vapeur de mercure de $500 \mathrm{~W}$, on retrouve la courbe 


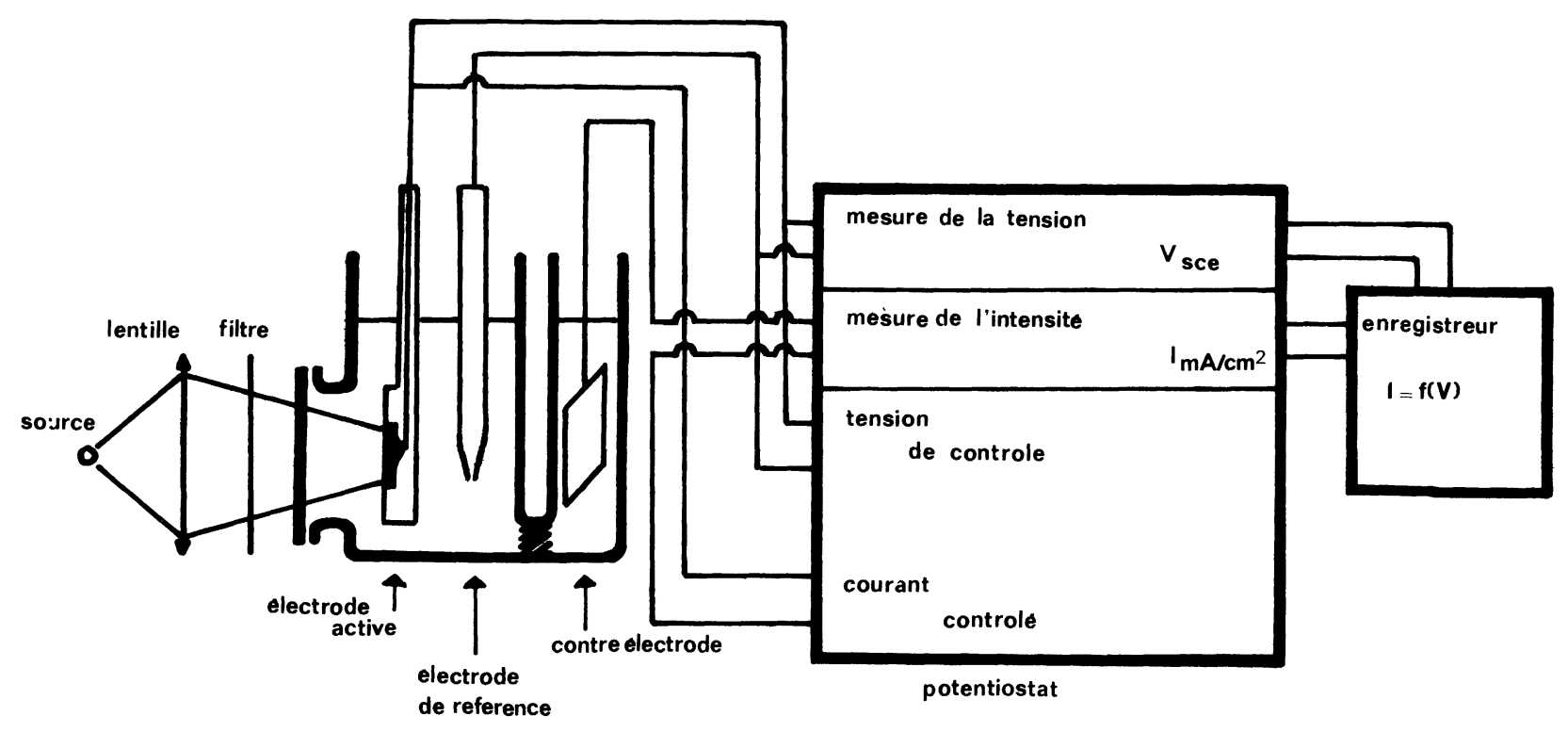

FIG. 1. - Montage potentiostatique d'étude de l'électrode.

[Experimental arrangement of the photoelectrochemical cell.]

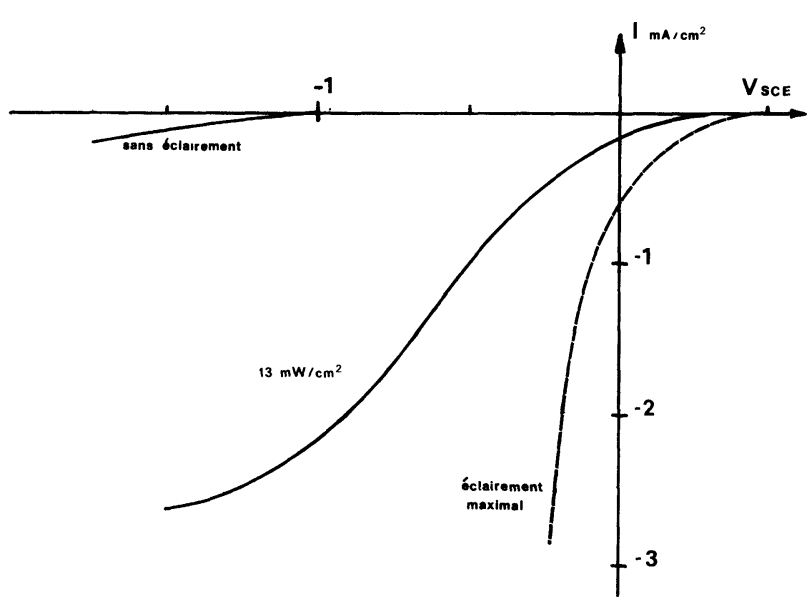

FIG. 2. - Courbes caractéristiques $I=f\left(V_{\text {sce }}\right)$ en polarisation cathodique de $\mathrm{GaP}$ p en milieu $\mathrm{SO}_{4} \mathrm{H}_{2}$ ou $\mathrm{HCl} 1 \mathrm{~N}$ : - dans le noir; - sous éclairement de $13 \mathrm{~mW} / \mathrm{cm}^{2}$; - sous éclairement très intense.

[Current-potential characteristics under cathodic polarization of p-GaP in $1 \mathrm{~N} \mathrm{SO}_{4} \mathrm{H}_{2}$ or $\mathrm{HCl}:$ - in the dark ; - under $13 \mathrm{~mW} / \mathrm{cm}^{2}$ illumination; - under very high illumination.]

observée par Yoneyama dans les mêmes conditions expérimentales : la tension $V_{\text {sce }}$ en circuit ouvert se déplace vers les potentiels positifs et les intensités sont nettement augmentées. Malgré cela sous une polarisation de $-1,5 V_{\text {sce }}$, l'intensité demeure stable durant plusieurs heures.

Réponse spectrale. La figure 3 indique l'intensité du courant traversant la cellule en milieu $\mathrm{HCl} 0,5 \mathrm{~N}$ sous une polarisation de $-1,2 V_{\text {sce }}$, en fonction de la longueur d'onde de la lumière tombant sur le monocristal. L'éclairement est de $5 \mathrm{~mW} / \mathrm{cm}^{2}$. Le maximum de la photoréponse se produit vers $4100 \AA$, c'est-àdire pour une énergie des photons incidents supérieure à l'énergie $E_{0}$ de la transition directe $(2,78 \mathrm{eV})$.

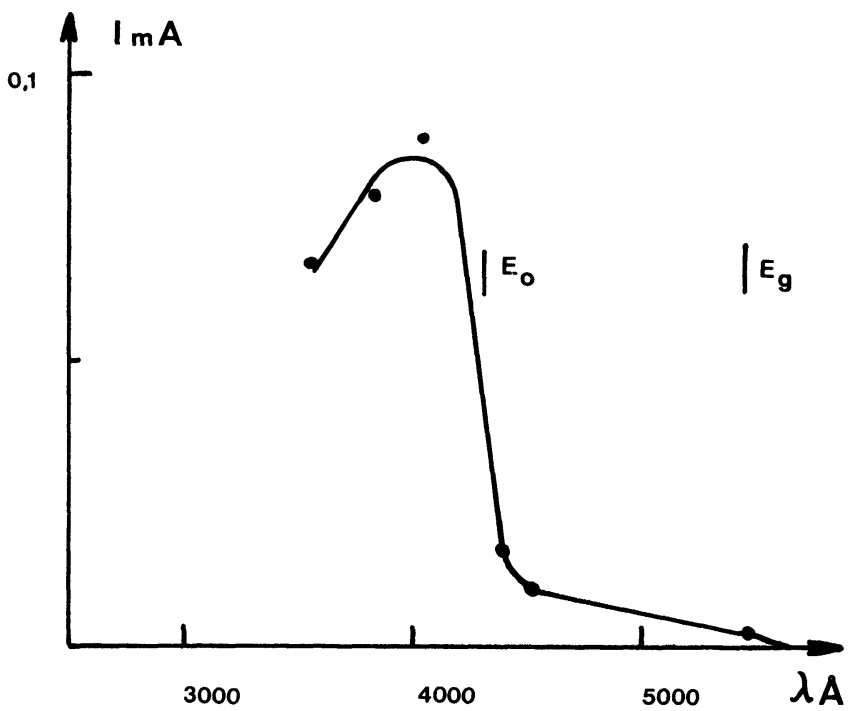

Fig. 3. - Réponse spectrale du GaP p sous polarisation de - 1,2 V. Puissance lumineuse monochromatique $5 \mathrm{~mW} / \mathrm{cm}^{2}$.

[Spectral response of $\mathrm{p}-\mathrm{GaP}$ under $-1.2 \mathrm{~V}$ polarization. The power density of the monochromatic light is $5 \mathrm{~mW} / \mathrm{cm}^{2}$.]

Lorsque l'énergie des photons incidents est comprise entre la largeur de bande interdite $E_{\mathrm{g}}=2,25 \mathrm{eV}$ et $E_{0}$, on n'observe qu'un faible photocourant. Dans ce domaine d'énergie, la transition de bande à bande est indirecte et la longueur d'absorption des photons est grande devant la largeur de la zone de charge d'espace, ces deux quantités ne devenant du même or̉dre de grandeur que pour des transitions directes, c'est-àdire pour des énergies supérieures à $E_{0}$.

Performances de l'électrode. Une évaluation des performances propres au système électrode-électrolyte peut être faite à partir des courbes intensité-potentiel par la mesure, pour une longueur d'onde lumineuse 


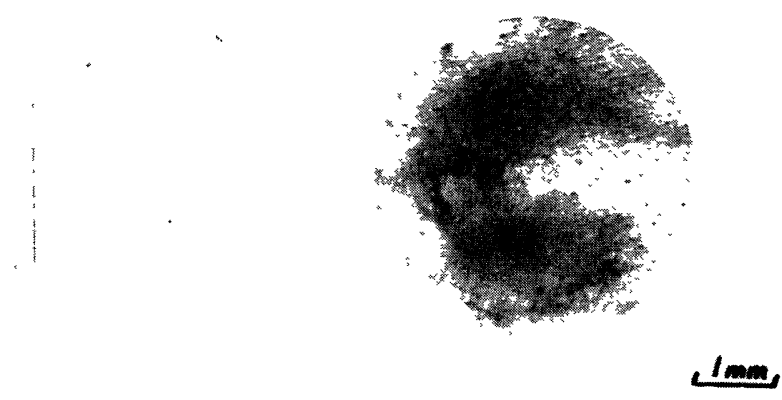

FiG. 4. - Aspect de la surface de l'électrode avant et après 10 heures de fonctionnement.

[Aspect of the electrode surface before and after 10 hours of working.]

donnée et sous la tension de saturation, du rapport de conversion photon-électron (nombre d'électrons extraits du semi-conducteur/nombre de photons incidents). Dans le cas du GaP p soumis à une densité de puissance lumineuse incidente de $13 \mathrm{~mW} / \mathrm{cm}^{2}$, pour une longueur d'onde de $3800 \AA$ et sous une polarisation de 1,5 $\mathrm{V} \mathrm{SCE}$, ce rapport atteind 60 $( \pm 10) \%$. La marge d'erreur observée est due à la médiocre reproductibilité des courbes intensité-potentiel signalée plus haut. Quoique important, ce rendement est sensiblement inférieur aux $100 \%$ prédits par Gerischer [4]. Il est possible que le niveau de dopage de notre échantillon $\left(2 \times 10^{18} \mathrm{Zn} / \mathrm{cm}^{3}\right)$ soit trop important. pour que l'absorption de la lumière se produise dans les conditions optimales.

Pile $\mathrm{TiO}_{2}$ n-GaP p. La valeur indiquée ci-dessus est le rendement maximum que pourrait atteindre cette demi-cellule associée à une autre demi-cellule pour produire de l'électricité ou transformer un système redox, si cette dernière permettait une polarisation adéquate. Si l'on compare (Fig. 5) les caractéristiques $i(V)$ du $\mathrm{TiO}_{2} \mathrm{n}[5,6,2]$ et du GaP p, on voit qu'il est possible de faire fonctionner une pile avec anode en $\mathrm{TiO}_{2} \mathrm{n}$ et cathode en $\mathrm{GaP} \mathrm{p}$ plongées dans un même électrolyte acide $(\mathrm{HCl} 1 / \mathrm{N})$ et de provoquer l'électrolyse de l'eau. Nous avons réalisé une telle pile, mais ses performances à plein éclairement sont très faibles, car on est amené à travailler en court-circuit entre les points $\mathrm{A}$ et $\mathrm{B}$. On est très loin des conditions optimales de polarisation de chaque électrode et les densités de courant n'excèdent pas $1 \mathrm{~mA} / \mathrm{cm}^{2}$. Le faible rendement de la pile $\mathrm{TiO}_{2} \mathrm{n}-\mathrm{GaP} \mathrm{p}$ en milieu acide $\left(\mathrm{H}_{2} \mathrm{SO}_{4} 0,2 \mathrm{~N}\right)$ a été observé par Nozik [7] qui l'a évalué à $0,25 \%$ sous un éclairement de $85 \mathrm{~mW} / \mathrm{cm}^{2}$.

Corrosion. La corrosion n'est apparue au microscope optique qu'après un fonctionnement sous éclairement direct à pleine puissance de la lampe à

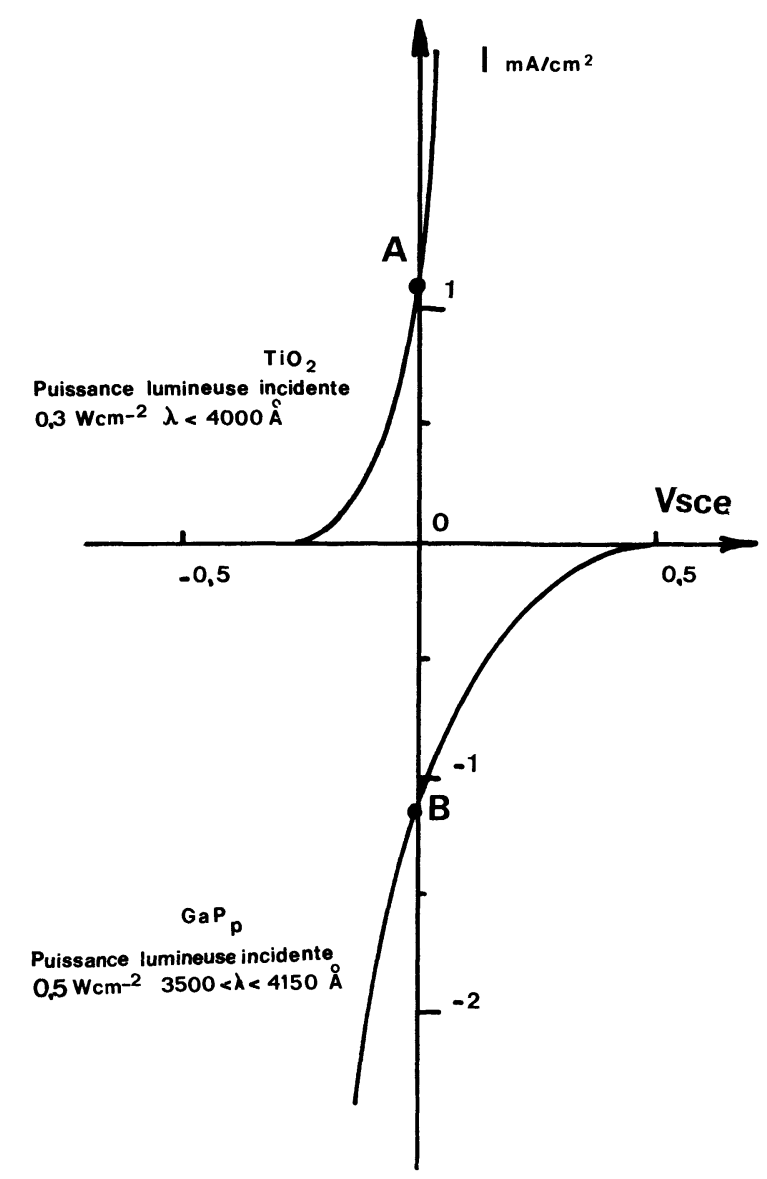

FIG. 5. - Comparaison entre les caractéristiques du $\mathrm{TiO}_{2}$ n polarisé anodiquement et du $\mathrm{GaP}$ p polarisé cathodiquement.

[Comparison between the current-potential characteristics of $\mathrm{n}-\mathrm{TiO}_{2}$ under anodic polarization and $\mathrm{p}-\mathrm{GaP}$ under cathodic polarization.]

vapeur de mercure de 500 We qui a duré 5 heures. La tache corrodée dessine la partie la plus lumineuse de l'image de la source sur la surface de l'échantillon. Cette tache est colorée en brun. La corrosion se manifeste principalement sur des défauts de surface (photo Fig. 4). Toutes les recherches que nous avons effectuées pour identifier le gallium ou le phosphore dans la solution, sont restées sans résultat.

4. Conclusions. - Le GaP de type p peut être utilisé comme cathode dans un dispositif photoélectrochimique. Cependant il n'utilise pas bien le spectre solaire puisque le maximum d'efficacité n'est obtenu qu'à la limite entre visible et U.V. Il serait intéressant d'utiliser un semi-conducteur dont la transition directe se situe à une énergie plus faible comme par exemple les composés GaInP ou GaAsP.

\section{Bibliographie}

[1] Beckmann et Memming, J. Electrochem. Soc. 116 (1969) 368.

[2] Yoneyama, SaKamoto, Tamura, Electrochim. Acta 20 (1975) 341.

[3] Gerischer, J. Electrochem. Soc. 113 (1966) 1174.

[4] GERISCHER, Electroanalytical chemistry and interfacial electrochemistry 58 (1975) 263.
[5] Fujischima, Honda, Kileuchi, Kagya Kagya Kagahu Zasshijap. 72 (1969) 108

[6] Desplat, J. Appl. Phys. 47 (1976) 5102.

[7] Nozıк, A. J., Appl. Phys. Lett. 29 (1976) 150. 